\title{
Transcatheter embolisation of an enlarging acquired coronary arteriovenous fistula in a heart transplant recipient
}

\author{
S C Eccleshall, M Pitt, J N Townend
}

\begin{abstract}
A 50 year old, recent cardiac transplant recipient developed systolic and diastolic murmurs but remained asymptomatic. The cause of the murmurs was not evident at transthoracic echocardiography. During routine left heart catheterisation a left anterior descending artery to right ventricular fistula was evident arising from the distal vessel and presumably acquired during routine endomyocardial biopsy. One year later, the patient remained asymptomatic but the calibre of the left anterior descending artery had increased and there appeared to be poor flow in to the proximal branches. The fistula was successfully treated by percutaneous deployment of two detachable embolisation coils in to the distal left anterior descending artery.
\end{abstract}

(Heart 1997;78:203-205)

Keywords: heart transplantation; transcatheter embolisation; arteriovenous fistula

A 50 year old man with heart failure owing to coronary artery disease underwent orthotopic heart transplantation in 1994. The procedure was successful and the patient was discharged from hospital 17 days after surgery on routine medical treatment including triple immunosuppressive therapy. His clinical course during the first year was uneventful without intercurrent infection or cellular rejection on routine endomyocardial biopsy. During this time he underwent 12 endomyocardial biopsies that were performed without apparent complication using a Harefield bioptome from the right internal jugular vein. Ten months after transplantation it was recorded that he was asymptomatic but a pansystolic murmur was noted at the apex and left sternal edge. There were no signs of cardiac failure and electrocardiography and chest radiography showed no new changes. Echocardiography showed no valve lesions and, although mild mitral and tricuspid regurgitation were present on Doppler interrogation, the cause of the murmur was unclear. At one year the patient remained asymptomatic without limiting dyspnoea or fatigue. On examination a continuous (systolic and diastolic) murmur was present without a thrill or other abnormal findings. He underwent a series of routine investigations including coronary angiography. This demonstrated good left ventricular function and no significant coronary arterial stenosis. A fistula between the distal left anterior descending (LAD) coronary artery and the right ventricle was noted with contrast medium passing freely into the right ventricle on opacification of the left coronary artery (fig 1). No action was taken. The patient remained well without limiting dyspnoea during the second post-transplant year.

Two years after transplantation, physical signs, electrocardiography, and chest radiography were unchanged. Coronary angiography was performed. Left ventricular function remained good with no significant change from the study after one year. There was no angiographic evidence of occlusive coronary artery disease. Direct comparison of the angiography videotapes from each study, 12 months apart, suggested that the calibre of the left anterior descending artery had increased (although no quantitative coronary angiography had been performed at the initial study), and the size of the shunt assessed subjectively by flow of contrast in to the right ventricle

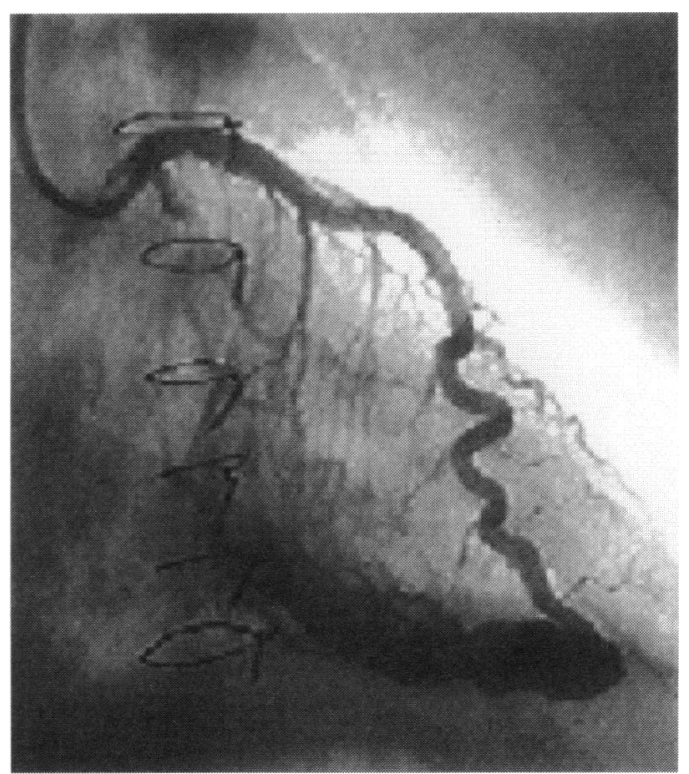

Figure 1 Angiography reveals the fistula between the left anterior descending coronary artery and the right ventricle. 


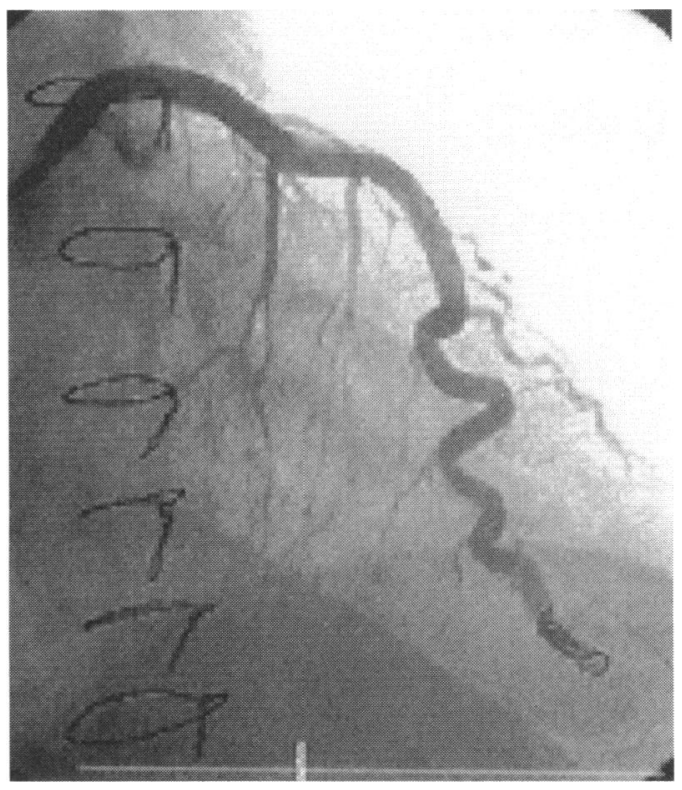

Figure 2 Angiography two hours after embolisation shows closure of the fistula with good opacification of the proximal vessel and branches.

appeared to have increased. There appeared to be poor flow in to the septal and diagonal branches of the LAD proximal to the fistula. The left to right shunt measured by oximetry was $1 \cdot 4: 1$. In view of these findings it was decided to close the fistula by transcatheter embolisation.

A guiding catheter ( 8 French, Judkins left 4) was positioned in the left coronary ostium and a 0.014 inch floppy guidewire was passed down the LAD and across the fistula in to the right ventricle. Over this wire a 3 French catheter was passed to the site of the fistula. Two detachable stainless steel coils each of $5 \mathrm{~mm}$ unconstrained diameter and $8 \mathrm{~cm}$ uncoiled length (Target Therapeutics, Fremont, California, USA) were then positioned in the distal LAD immediately above the fistula. The procedure was uncomplicated. Flow in to the right ventricle was immediately diminished. A repeat study, two hours later, showed complete occlusion of the fistula with good opacification of the proximal LAD and branches (fig 2). Following the procedure the murmur disappeared, there were no changes in the electrocardiogram, and no rise in cardiac enzymes was detected.

\section{Discussion}

Acquired coronary artery fistulae as a result of endomyocardial biopsy are a frequently observed feature of coronary angiograms of heart transplant recipients. The incidence has been estimated to be as high as $\mathbf{8 \%}$, although more recent reports suggest a figure of $3 \%^{12}$ and, as in the present case, they almost always drain in to the right ventricle from the LAD. Presumably an epicardial vessel is damaged as a result of the biopsy procedure; pericardial fat containing small arteries is a not infrequent finding in endomyocardial biopsy samples from heart transplant patients. The pericardial space in heart transplant patients is obliterated and fistula formation rather than tamponade is the most common complication of damage to pericardial vessels. Review of all the biopsies from our patient failed to identify any unusual features, no sample contained tissue from a major arterial wall.

The natural history of such fistulae has not been studied in large numbers of patients but available information suggests that problems seldom occur. In 17 fistulae in 14 heart transplant patients followed for a median of six years, the majority ( $71 \%$ ) closed spontaneously, none increased in size, and no clinical complications occurred. ${ }^{2}$ It was this information that prompted our wait and see management after one year. A search of the literature has revealed only three cases that required closure, ${ }^{3-5}$ all because of symptoms of dyspnoea and fatigue apparently related to the fistula. However, in two of these cases there were multiple fistulae between the $\mathrm{LAD}$ and right ventricle. Our case demonstrates that even single biopsy related fistulae are not invariably benign; we documented an increase in the size of the fistula over a period of only one year. Despite the absence of symptoms, we felt that closure was indicated in this patient who appeared to be developing a high flow fistula progressively dilating the $\mathrm{LAD}$ and causing steal from the LAD branches. Late complications of other acquired coronary artery fistulae (due to trauma) in non-transplant patients include angina, heart failure, and atrial arrhythmias. 6 Persistence of, or increase in, flow has been suggested as an indication for early closure.

The method of closure depends on the individual anatomical features of fistulae. Transcatheter closure has been used widely in paediatric cases of congenital coronary arteriovenous fistula and less frequently in adults. ${ }^{78}$ We have found one report of open surgical closure of a coronary artery fistula in a transplant patient ${ }^{5}$ and two reports of transcatheter closure; both of these were multiple fistulae draining in to the right ventricle from the LAD. ${ }^{34}$ In our case, the distal LAD immediately above the single distal fistula provided an ideal position for coil placement without jeopardising other branches.

This case demonstrates that despite earlier reports of their benign course, acquired coronary arteriovenous fistulae in heart transplant recipients can progressively increase in size, causing steal from proximal branches, and risking late complications. Transcatheter embolisation with detachable coils appears a safe and effective treatment option.

We gratefully acknowledge the assistance of $\mathrm{Mr} \mathrm{R} S$ Bonser, $\mathrm{Dr}$ N P Buller, Dr R West, and Dr A Howie.

1 Sandhu JS, Uretsky BF, Zerbe TR, Goldsmith AS, Reddy S, Kormos RL, et al. Coronary artery fistula in the hear transplant patient. A potential complication of endomyocardial biopsy. Circulation 1989;79:350-6.

2 Lazar JM, Uretsky BF. Coronary artery fistula after heart transplantation: a disappearing entity? Cathet Cardiovasc transplantation: a disappear

3 Furniss SS, Mitchell L, Kitipawong P. Coil embolization of a coronary fistula in a post transplant patient. Eur Heart $\mathcal{f}$

4 Fontanelli A, Gasparini D, Morocutti G, Di Chiara A, 
Feruglio GA. La chiusura percutanea delle fistole arteriose coronariche congenite e iatrogene. G Ital Cardiol 1995;25:1381-7.

5 Uchida N, Baudet E, Roques X, Laborde N, Billes MA. Surgical experience of coronary artery-right ventricular fistula in a heart transplant recipient. Eur $¥$ Cardiothorac Surg 1995;9:106-8.

6 Shimabukuro $M$, Shinzato $T$, Yoshida $H$, Nagamine F, Takasu N, Koja K. Late complications in a traumatic coronary artery fistula; a report of a case requiring surgical repair after 8 years. Cardiology 1996;87:86-9.

7 Qureshi SA, Reidy JF, Bin Alwi M, Lim MK, Wong J, Tay J, et al. Use of interlocking detachable coils in embolization of coronary arteriovenous fistulas. $A m \mathcal{f}$ Cardiol 1996; 78:110-13.

8 Perry SB, Rome J, Keane JF, Baim DS, Lock JE. Transcatheter closure of coronary artery fistulas. $\mathcal{F} \mathrm{Am}$ Coll Cardiol 1992;20:205-9.

\section{Single coronary artery with infundibular pulmonary stenosis}

Taku Yamamoto, Yoshizumi Habuchi, Junichiro Morikawa

Third Department of Internal Medicine, Kyoto Prefectural University of Medicine, KawaramachiHirokoji, Kamigyo-ku, Kyoto 602, Japan $T$ Yamamoto J Morikawa Department of Laboratory Medicine Y Habuchi

Correspondence to: Dr Yamamoto. 28 May 1997 Accepted for publication

Single coronary artery is a rare congenital anomaly. ${ }^{1}$ Infundibular pulmonary stenosis (IPS) without ventricular septal defect or tetralogy of Fallot (isolated IPS) is also very rare. ${ }^{2}$ We report a case of single coronary artery with isolated IPS; to our knowledge, this is the first report of such a case.

\section{Case report}

A 57 year old asymptomatic man was referred to our hospital because of chest radiography and ECG abnormalities. Physical examination revealed a grade 4 harsh systolic ejection murmur heard best in the fourth intercostal space at the left sternal border. Chest radiography showed right atrial and ventricular enlargement with a cardiothoracic ratio of 0.55 . Pulmonary vascularity was normal. ECG showed right ventricular hypertrophy.

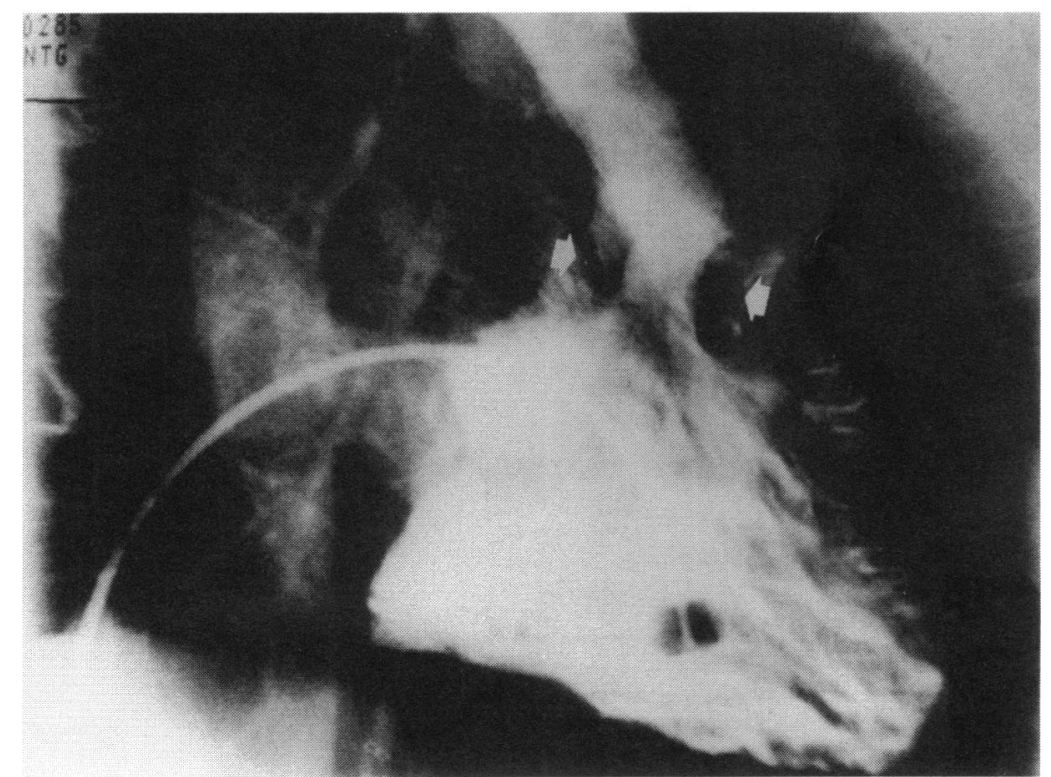

Figure 1 Right anterior oblique view of right ventricular angiography revealing infundibular pulmonary stenosis (arrows). Both the pulmonic valve and the ventricular septum were intact.
Echocardiography revealed a dilated right ventricular body and a protruding lesion at the proximal portion of the infundibulum. Cardiac catheterisation disclosed a peak systolic pressure gradient of $68 \mathrm{~mm} \mathrm{Hg}$ between the right ventricular body and the infundibulum. The right atrial and the pulmonary arterial pressures were normal. Oxygen sampling revealed no evidence of shunting. Right ventricular angiography revealed a prominent muscle band at the junction of the right ventricular body and the infundibulum (fig 1). Both the pulmonic valve and the ventricular septum were intact. Coronary artery angiography revealed a solitary left sided coronary ostium. An anomalous right coronary artery ran from the left anterior descending artery anteriorly to the root of the main pulmonary artery without stenosis (fig 2). It arose from the middle portion very close to the first septal perforating branch of the left anterior descending artery. These findings are compatible with isolated IPS associated with single coronary artery.

The patient underwent surgical resection of the anomalous muscle band and no vestige of ventricular septal defect was found at operation.

\section{Discussion}

In most cases of single left coronary artery, the right coronary artery arises from the main trunk, the proximal portion of the left anterior descending artery, or the left circumflex artery. ${ }^{3}$ Few reports have described cases of anomalous right coronary artery arising from the middle portion of the left anterior descending artery without other congenital anomalies. ${ }^{13}$ Single coronary artery is usually combined with various cardiac anomalies such as improper division of truncus arteriosus, transposition of great vessels, and tetralogy of Fallot. The present case was associated with isolated IPS, itself a rare anomaly. Most patients with IPS are diagnosed and surgically 